\title{
Regularized full-waveform inversion for large 3-D salt bodies
}

Mahesh Kalita ${ }^{l}$, King Abdullah University of Science and Technology, Ahmad Riza Ghazali ${ }^{2}$, Petroliam Nasional Berhad (Petronas), Kefeng Xin ${ }^{2}$, Farah Syazana Dzulkefli ${ }^{2}$ and Tariq Alkhalifah ${ }^{1}$

\section{SUMMARY}

Our objective is to invert for large 3-D salt models using fullwaveform inversion (FWI), especially in the absence of good starting models and low frequencies in the seismic data. This objective incurs a couple of critical issues. First, the presence of salt geobodies aggravates the non-linearity and illposedness of FWI. Second, a 3-D FWI is computationally very expensive, even more so when the initial model is in hindsight very far from the target one. To mitigate the ill-posedness, we propose to utilize model regularization in the FWI framework to promote a limited variation in the inverted model followed by a post-processing step of FWI to penalize sharp velocity drops in the model. Next, to reduce the computational overburden, we propose to utilize a multi-excitation assumption (MExA) of source wavefields in the FWI gradient calculation step. This assumption, due to the simplistic nature of the source wavelet, approximates the source wavefield wiggle at a gridpoint by a series of its energetic arrivals. As a result, the gradient evaluation using MExA requires us neither to store the entire source wavefield nor to include an additional extrapolation step to propagate the source wavefield from its temporary storage at the boundary. The versatility of the proposed method is demonstrated on a synthetic dataset of the modified SEG/EAGE salt model in which the lowest available frequency is $3 \mathrm{~Hz}$.

\section{INTRODUCTION}

Despite the continued advancements of full-waveform inversion (FWI) in the recent years (Tarantola, 1984; Virieux and Operto, 2009), its theoretical foundation, which is based on the single scattering assumption, known as the Born approximation, often break down in the absence of low frequencies from recorded seismic data given the initial model is very far from the true subsurface model. This limitation of FWI often gets aggravated particularly in the presence of models with high-velocity structures over a wide region, like salt bodies and the sediments beneath them. As a result, FWI fails to yield an adequate representation of the subsurface model. That is why it takes a backseat whenever a salt province is under consideration, particularly in the absence of a good starting model. In that case, the manually driven 'migrate-pick-flood' approach remains a preferable, albeit erroneous, industrial solution to build the velocity model. However, several FWI studies based on the level-set method (Lewis et al., 2012; Kadu et al., 2017), novel objective functions (Yang and Engquist, 2017; Wu and Chen, 2018), model regularization (Esser et al., 2016; Brandsberg-Dahl et al., 2017) and so on, have been proposed in recent years as an alternative to the above mentioned industrial ad-hoc practice. Towards that aim, this article extends the idea proposed in Kalita et al. $(2018 \mathrm{a}, \mathrm{b})$ to a 3-D regime. That is, we formulate the minimization problem into two nested subproblems: first, we attempt to minimize the data mismatch and the total variation in the model; second, we minimize sharp velocity drops with depth in the model. This second step results in a flooding of high velocities from the top of the salt (without picking), obtained from the first step of our proposed formulation. This split approach is extremely convenient because it is easy to implement into the existing FWI machinery. Moreover, multiple regularization weights can be efficiently tested in the flooding process.

The success of FWI relies heavily on the initial model or/and the available low-frequency bandwidths of the seismic data. Those conditional requirements are relevant, even more so to a large 3-D salt province due to its size. For example, Shen et al. (2018) reported substantial improvements in the subsalt legacy model of the Atlantis region by using a newlyrecorded dataset containing frequency as low as $1.6 \mathrm{~Hz}$. However, in general, various factors including instrumental limitations, system noise and so on prevent the receivers from recording low-frequency signals with acceptable signal-to-noise ratio. In that case, a wide azimuth long-offset acquisition survey becomes an alternative to the low-frequency information of the data in the context of retrieving long wavelelength features, especially of the subsalt region. However, in this case, we usually pay the price with a significant increase in the computational cost. Since FWI aims to delineate the fine-scale details of the salt province including steeply dipping flanks and subsalt sharp anomalies, the inversion requires a fine numerical grid in the model. As a consequence of the CourantFriedrichs-Lewy condition, the salt model because of highvelocity zone imposes even a smaller time sampling in the seismic data. Concurrently, the need for more sophisticated algorithms to ameliorate the prevalent concerns in a wide azimuth data particularly the cycle skipping and anisotropy, results in additional computational costs in FWI. In a summary, these factors lay down computational challenges to make FWI work for a 3-D salt model. The excitation method in this regard is promising as it reduces the computational requirements to a feasible level.

The excitation approach (ExA) utilizes the most energetic part of the source wavefield in order to compute the gradient (Kalita and Alkhalifah, 2017). Most of the energy to the gradient computation is usually extracted from a single scattering optimal (energy wise) path of the waves. However, it might delay the recovery process, especially of salt-effected seismic data enriched by strong multiples. That provides the motivation to accommodate more arrivals in the gradient calculation step under the ExA framework, without hampering its computational benefits. It requires neither to save the entire source wavefield history nor the additional modelling step of boundary saving scheme. This article proposes multi-excitation approximation (MExA) to calculate the 3-D FWI gradient more effi- 


\section{FWI for 3-D salt bodies}

ciently than the conventional counterparts. The remaining part of this article proceeds as follows. We begin the theory section on FWI with model regularization, which retrieves the sharp edges of the salt body and subsequently, floods it. Next, we report a short review of ExA proposed in Kalita and Alkhalifah (2017), followed by a straightforward extension of ExA to incorporate more than a single arrival in the gradient computation procedure. To demonstrate the versatility of the proposed method, we consider a modified version of the SEG/EAGE salt model, a Ricker wavelet of dominant frequency $5.0 \mathrm{~Hz}$ and minimum frequency 3.0 to synthesize the observed seismic data. We begin the inversion process with a very poor model that contains no information of the salt body. The proposed methodology successfully retrieves most, if not all of the target features, whereas the traditional FWI completely fails.

\section{THEORY}

The absence of low frequencies in the seismic data often steers the gradient evaluation, especially beneath the top of the salt, in the wrong direction. As a result, FWI renders an undesirable low-velocity field in that region, which prevents the inversion from recovering the deeper events including the bottom of the salt and the subsalt regions. To mitigate this issue, we propose to apply regularized FWI with automated flooding as mentioned in the following section.

\section{Regularized FWI with automated flooding}

Similar to Kalita et al. (2018b), we have decoupled the problem into two parts as follows:

\section{PROBLEM 1 : FWI+TV}

$$
J_{F W I+T V}(\mathbf{m})=\min _{\mathbf{m}}\left\{\left\|\mathbf{d}_{o}-\mathbf{d}_{m}\right\|_{2}^{2}+\lambda_{T V}\|\mathbf{m}\| \|_{T V}\right\}
$$

with $\mathbf{d}_{0}$ : observed data; $\mathbf{d}_{m}$ : predicted data; $\|\mathbf{m}\|_{T V}=$ $|\nabla \mathbf{m}|=\sqrt{\mathbf{m}_{x}^{2}+\mathbf{m}_{y}^{2}+\mathbf{m}_{z}^{2}}$ is the TV regularization.

PROBLEM 2 : FLOODING

$$
J_{F}(\mathbf{q})=\min _{\mathbf{q}}\left\{\|\mathbf{q}-\mathbf{u}\|_{2}^{2}+\lambda_{F}\|\mathbf{q}\|_{F}\right\}
$$

with $\mathbf{u}: \underset{\mathbf{m}}{\operatorname{argmin}} J_{F W I+T V} ; \quad\|\mathbf{q}\|_{F}$ : flooding regularization defined as follows

$$
\|\mathbf{q}\|_{F}=\frac{1}{2 p}\left\|\left(\left|\mathbf{q}_{z}\right|-\mathbf{q}_{z}\right)^{p}\right\|_{2}^{2} \quad \text { with } \quad p>1 / 2
$$

$\lambda_{T V}, \lambda_{F} \geq 0$ are the regularization coefficients of the TV and flooding regularization terms. The first problem, 'FWI+TV' aims to minimize the data misfit and the total variation of the model. In this way, it recovers the velocity field by reinforcing the sharp edges of the salt structure without over-smoothing them. The second problem, 'flooding' penalizes the velocity drops with depth in the reconstructed model using 'FWI+TV', The gradient direction of flooding regularization is defined as:

$$
\frac{\delta\|\mathbf{q}\|_{F}}{\delta \mathbf{q}}= \begin{cases}-(2 p-1) 4^{p} \mathbf{q}_{z}^{2(p-1)} \frac{\partial \mathbf{q}_{z}}{\partial z} & \text { for } \mathbf{q}_{z}<0 \\ 0 & \text { otherwise }\end{cases}
$$

Equation (3) suggests that the gradient direction of the flooding regularization mainly depends on the quantity $-\mathbf{q}_{z}^{2(p-1)} \frac{\partial \mathbf{q}_{z}}{\partial z}$. A careful observation of this quantity reveals that the parameter $p>1$ favors the gradient direction towards a region of steeper velocity drops, whereas a value of $1 / 2<p \leq 1$ produces an impartial gradient by setting $\mathbf{q}_{z}^{2(1-p)}$ as a denominator in equation (3). Towards the goal of removing that undue low-velocity zone without hampering the rest of the model in the outcome of 'FWI+TV', we require to consider $p>1$ in the 'flooding' problem. In this study, we set $p=2$.

\section{Computation of FWI gradient based on MExA}

Limited computer resources impair the proposed framework of Kalita et al. (2018b) from delivering its promise of yielding salt-provinces from 3-D seismic data. To ameliorate the computational overburden, we propose to apply the excitation assumption to compute the FWI gradient. This section sheds some light on the excitation method proposed in Kalita and Alkhalifah (2017) followed by its adaption to compute the data misfit gradient of salt-affected seismic dataset.

According to Nguyen and McMechan (2013), the ExA simplifies the entire source wavefield, $\mathbf{u}$, at a gridpoint $\mathbf{x}$ by its most energetic amplitude and arrival time, known as excitation amplitude and excitation time $\left(t_{e x}\right)$, respectively. Mathematically, excitation wavefield, $\mathbf{u}_{\delta}$, resulting from a source placed at $\mathbf{x}_{s}$ is a scaled impulse defined as $\mathbf{u}_{\delta}\left(\mathbf{x}, t ; \mathbf{x}_{s}\right)=\mathbf{u}\left(\mathbf{x}, t ; \mathbf{x}_{s}\right) \delta\left(t-t_{e x}\right)$. Based on this assumption, Kalita and Alkhalifah (2017) evaluates the gradient direction as follows:

$$
\mathbf{g}_{e x}=-\mathbf{u}_{\delta}^{T}\left(\mathbf{L}^{-1}\right)^{T} R^{T} \mathbf{F}^{*} \delta \mathbf{s}
$$

where $\delta \mathbf{s}$ is the adjoint-source; $\mathbf{F}^{*}$ is an upper triangular Toeplitz matrix with $j^{\text {th }}$ column given by

$\left(\left.\frac{\partial^{2} f}{\partial t^{2}}\right|_{t_{j}},\left.\left.\frac{\partial^{2} f}{\partial t^{2}}\right|_{t_{j-1}} \quad \ldots \quad \frac{\partial^{2} f}{\partial t^{2}}\right|_{t_{2}},\left.\quad \frac{\partial^{2} f}{\partial t^{2}}\right|_{t_{1}} \quad \ldots 0\right)$ that acts on $\delta \mathbf{s}$ representing the temporal cross-correlation process. In other words, equation 4 states (from right to left) that the adjoint source is to be cross-correlated with the second order time-derivative of the source wavelet and back-propagated in time from the position of receivers $\left(R^{T} \mathbf{F}^{*} \delta \mathbf{s}\right)$, using an operator $\mathbf{L}^{T}$ followed by a temporal zero-lag cross-correlation with the excitation wavefield, $\mathbf{u}_{\delta}$, to obtain the excitation-based FWI gradient. Since $\mathbf{u}_{\delta}$ at a grid point $\mathbf{x}$ contains only one non-zero arrival, equation 4 can be simplified to

$$
\begin{array}{r}
g_{e x}(\mathbf{x})=-\mathbf{u}_{\delta}\left(\mathbf{x}, t_{e x}(\mathbf{x})\right) \mathbf{v}\left(\mathbf{x}, t_{e x}(\mathbf{x})\right) \\
\text { with } \quad \mathbf{v}=\mathbf{L}^{*} \delta \hat{s} \text { and } \delta \hat{s}=\mathbf{F}^{*} \delta s,
\end{array}
$$

where $\delta \hat{s}$ represents the modified adjoint source, and $\mathbf{v}$ the back-propagated adjoint wavefield at a grid point $\mathbf{x}$. Therefore, equation 5 suggests the gradient evaluation based on ExA requires a storage of the excitation maps of the order of only the model size instead of the entire source wavefield history. Unlike the boundary-saving scheme, the ExA does not require any additional modelling steps to compute the gradient. However, this underpinning assumption of sparsity on the source wavefield does not hold well in presence of complex salt-environments. Consequently, the gradient computation based on ExA deprives us of a significant amount of multi scattering energy. As a result, the retrieval of the structure, especially the top of the salt, 


\section{FWI for 3-D salt bodies}

using ExA is much slower compared to that using conventional FWI. For that matter, we relax the strict definition of ExA to expedite the inversion process, and we include more energetic non-zero arrivals in the source wavefield. This way, we allow more arrivals of the seismic recordings to participate in the gradient computation step. In that case, the gradient computation is a straightforward extension of equation 5 given by:

$$
\begin{gathered}
\tilde{g}(\mathbf{x})=-\sum_{\text {count }=1}^{n} \mathbf{u}_{\delta}\left(\mathbf{x}, t_{\text {ex }}^{\text {count }}(\mathbf{x})\right) \mathbf{v}\left(\mathbf{x}, t_{\text {ex }}^{\text {count }}(\mathbf{x})\right) \\
\text { with } \mathbf{v}=\mathbf{L}^{*} \delta \hat{s} \text { and } \delta \hat{s}=\mathbf{F}^{*} \delta s
\end{gathered}
$$

where $n$ is the number of energetic arrivals of considerations.

\section{NUMERICAL EXAMPLE}

We demonstrate an application of the proposed method to a modified version of the SEG/EAGE salt model (Aminzadeh et al., 1997). The original model covers an area of $13.5 * 13.5$ square $\mathrm{km}$ horizontally and $4.0 \mathrm{~km}$ vertically. However, we adjust the size of the $y$ axis to half of its original size to reduce the number of gridpoints. This study considers a 3-D region of the size of $3.75 * 12.0 * 5.0 \mathrm{~km}$ from the modified version of original model (Figure 1). It contains a simple background with a complex salt structure with variable size and shape.

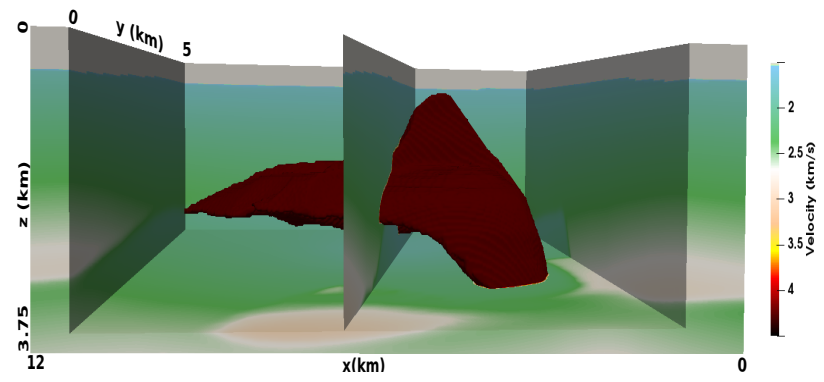

Figure 1: The modified SEG/EAGE salt model. We downscale the $y$ axis of the original model by a factor of two in order to reduce the number of gridpoints. The salt region is depicted by the $4.0 \mathrm{~km} / \mathrm{s}$ iso-velocity-surface.

\section{Acquisition Geometry}

The acquisition survey includes four streamers of length 12.0 $\mathrm{km}$ at the locations of $\mathrm{y}=\{1,2,3,4\} \mathrm{km}$ to record seismic gathers for 8 seconds. There are 2294 sources placed between between $(x, y)=(0.3,0.3)$ and $(11.4,4.8) \mathrm{km}$ at intervals of $0.150 \mathrm{~km}$ in this synthetic experimental setup. A Ricker wavelet of $5.0 \mathrm{~Hz}$ dominant frequency filtered by a low-cut filter at $3.0 \mathrm{~Hz}$, is used to synthesize the observed data with the free surface boundary condition. Figure 2 displays a shot gather from the source placed at $(x, y)=(6.0,2.7) \mathrm{km}$ on the surface and its frequency content, respectively.

\section{Inversion setup}

We extend the 2-D slice at $x=0 \mathrm{~km}$ of the target model (Figure 1) in the third dimension followed by a multidimensional triangular smoothing to obtain the initial model as shown in Figure 3. It contains no information of the salt. Moreover,

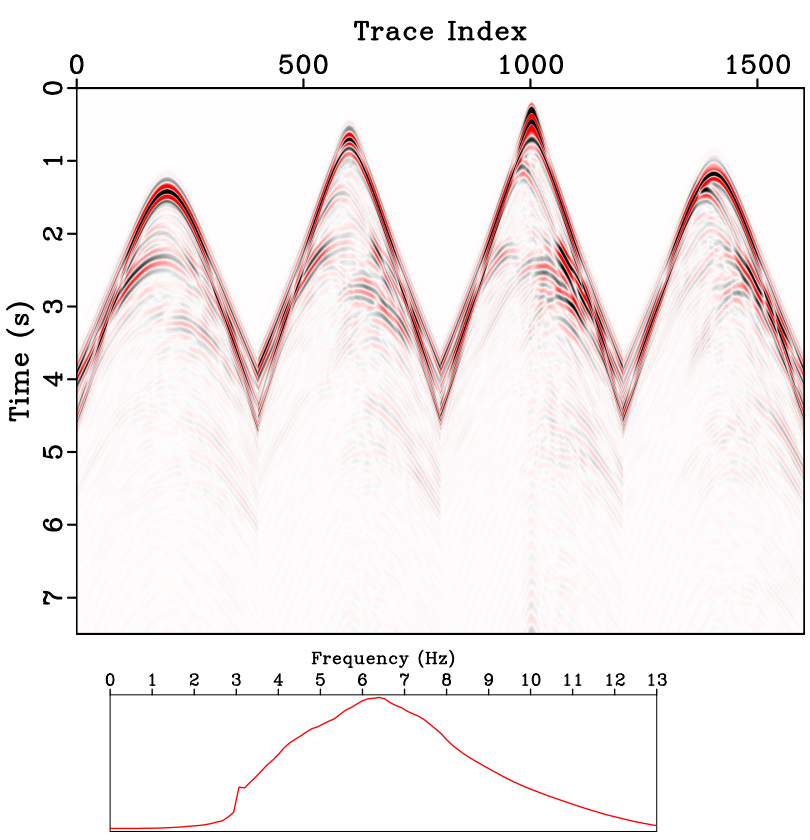

Figure 2: A shot gather from the source placed at $(x, y)=$ $(6,0,2.7) \mathrm{km}$ (top) and its average frequency spectrum (bottom).

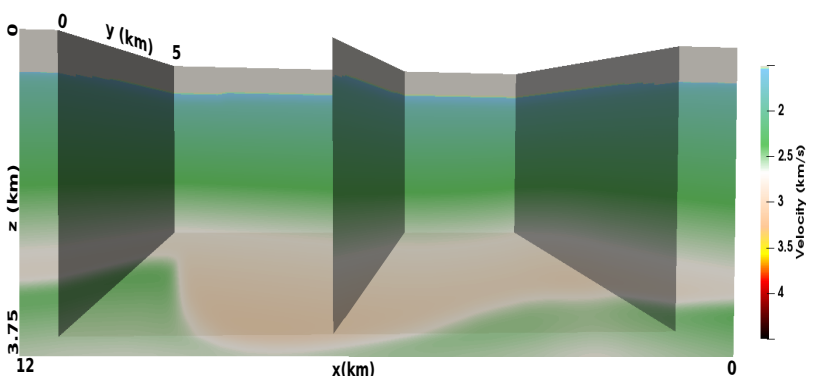

Figure 3: Initial model without any high-velocity zone.

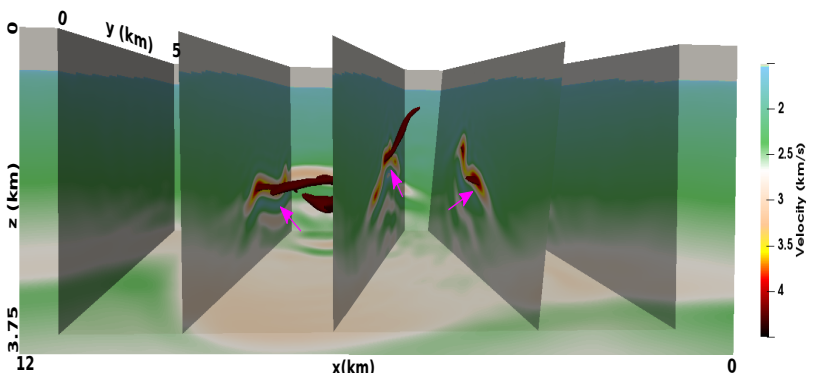

Figure 4: Output of the 'FWI+TV' stage. It attempts to reconstruct the top of the salt body. However, regions beneath the top of the salt as indicated by the arrows, recover wrong velocities. 


\section{FWI for 3-D salt bodies}

it has different sedimentary velocities compared to that of the target model. At every iteration of FWI, we randomly consider only 400 out of 2294 shot gathers. In this example, we consider five arrivals to execute MExA. Also, we follow the popular hierarchical, multiscale strategy (Bunks et al., 1995), starting the inversion at the lower frequency bandwidth and gradually including the higher frequencies. As the inversion makes progress, we decrease the regularization coefficients, and finally set them to zero. In this way, we reinforce the dominance of the seismic data in the reconstruction process over the regularization terms. As a result, the proposed methodology attempts to yield a model which is less biased by the initial values of the regularization parameters.

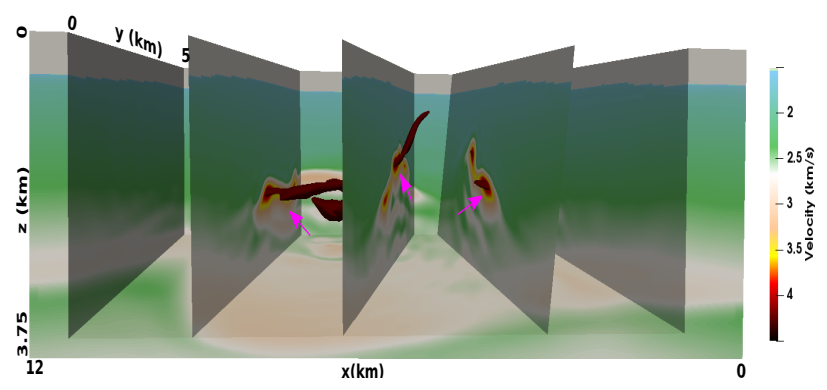

Figure 5: Output of the 'flooding' procedure with the input velocity from Figure 4 . The arrows indicate the regions where the 'flooding' succeeded in wiping out the wrong velocity of the reconstructed model of 'FWI+TV' (Figure 4).

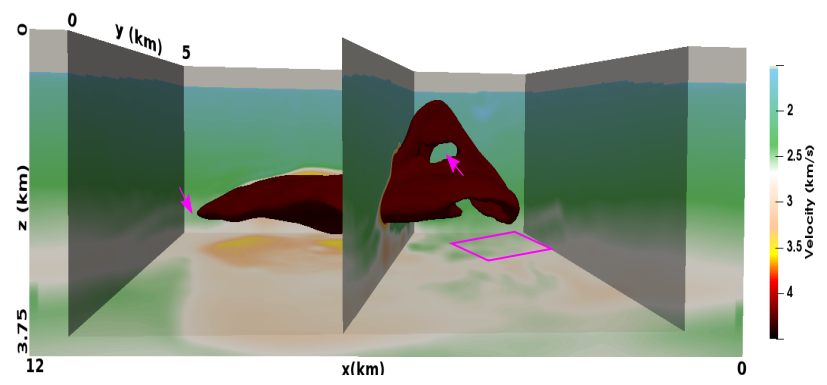

Figure 6: Final reconstructed model of 'FWI+TV' with $\lambda_{T V}=0$ for the full bandwidth of the dataset. The proposed method retrieves most, if not all of the target model (Figure 1). The regions indicated by the arrows and the rectangle fall sort of the velocity of the $4.0 \mathrm{~km} / \mathrm{s}$ iso-velocity-surface.

\section{Inversion results}

Figure 4 shows the output of 'FWI+TV' for the first bandwidth of the seismic data. As expected, the inversion attempts to recover the top of the salt. However, it fails to recover highvelocity regions just beneath the top of the salt as indicated by the arrows in Figure 4. This model is subsequently subjected to the 'flooding' procedure. It manages to remove the undesirable low-velocity zone as shown in Figure 5. Next, we consider this velocity model to stimulate the 'FWI+TV' scheme. It helps the model get rid of the unnecessary flooding residual if any. The final outcome of this stage is once again fed back into the flooding scheme. This feeding back system complements both subproblems and slowly starts retrieving the complex rugose

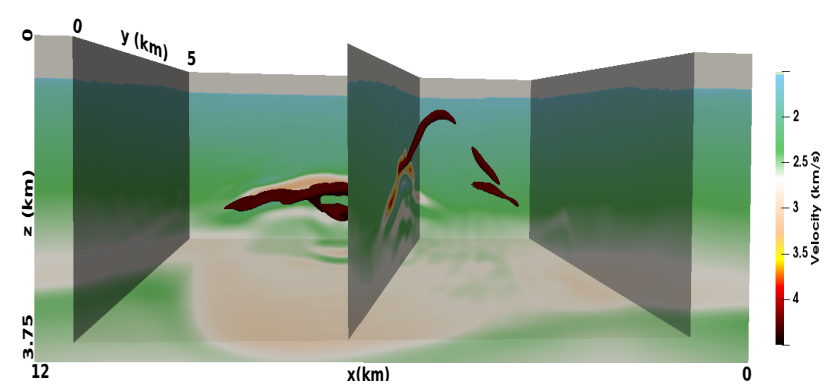

Figure 7: Final Reconstructed model using traditional FWI with no regularization. It severely fails to delineate the geological features of the target model.

salt features. For instance, the inversion scheme first reconstructs the top of the salt correctly, followed by the vicinity of the salt. After recovering the salt region, it focuses more on the recovery of subsalt, given the data of consideration provides favorable conditions including low frequency, wide azimuth, long-offsets. We display the final inverted model in Figure 6. It recovers most of the model, especially the salt structure. However, the inversion could not manage to recover all as pointed out by the arrows and the rectangular box in Figure 7. On the other hand, the traditional approach suffers a complete failure in the reconstruction process.

\section{CONCLUSIONS}

We have attempted to reconstruct 3-D salt bodies from recorded seismic data. To do so, we split the minimization problem into two subproblems, namely 'FWI+TV' and 'flooding'. The 'FWI+TV' promotes condensed variations in the model obtained from the data misfit function. The 'flooding' smears the high velocity vertically in a controlled manner wherever there is a drop of velocity with depth. Any undesired flooding will be eradicated by the data misfit term in the subsequent 'FWI+TV' stage of our cascading strategy. In order to reduce the computational overburden, we have computed the FWI gradient using the multi-excitation approximation method. Our proposed method is mathematically more robust and less cumbersome than the standard erroneous 'migrate-pick-flood' approach. We have shown the application of the proposed method to parts of the modified SEG/EAGE salt model, in which the lowest available frequency of the observed synthetic dataset is $3 \mathrm{~Hz}$. We also plan to present the results of the SEAM phase I subsalt model (downscaled version) in the annual meeting.

\section{ACKNOWLEDGMENTS}

We would like to thank KAUST and Petroliam Nasional Berhad (Petronas) for their support, all the members of Seismic Wave Analysis Group for fruitful discussions, the Supercomputer laboratory and IT Research Computing for computer time, the Core Labs of KAUST for their facilities. 


\section{REFERENCES}

Aminzadeh, F., J. Brac, and T. Kunz, 1997, 3-D salt and overthrust models, SEG/EAGE 3-D modeling series no.1: Society of Exploration Geophysicists.

Brandsberg-Dahl, S., N. Chemingui, A. Valenciano, J. RamosMartinez, and L. Qiu, 2017, Fwi for model updates in largecontrast media: The Leading Edge, 36, no. 1, 81-87.

Bunks, C., F. M. Saleck, S. Zaleski, and G. Chavent, 1995, Multiscale seismic waveform inversion: Geophysics, 60, no. 5, 1457-1473.

Esser, E., L. Guasch, F. J. Herrmann, and M. Warner, 2016, Constrained waveform inversion for automatic salt flooding: The Leading Edge, 35, no. 3, 235-239.

Kadu, A., T. van Leeuwen, and W. A. Mulder, 2017, Salt reconstruction in full-waveform inversion with a parametric level-set method: IEEE Transactions on Computational Imaging, 3, no. 2, 305-315.

Kalita, M., and T. Alkhalifah, 2017, Efficient full waveform inversion using the excitation representation of the source wavefield: Geophysical Journal International, 210, no. 3, 1581-1594.

Kalita, M., V. Kazei, Y. Choi, and T. Alkhalifah, 2018a, Fullwaveform inversion for automated salt flooding: Presented at the 80th EAGE Conference and Exhibition 2018.

$\longrightarrow, 2018 \mathrm{~b}$, Regularized full-waveform inversion for salt bodies, in SEG Technical Program Expanded Abstracts 2018: Society of Exploration Geophysicists, 1043-1047.

Lewis, W., B. Starr, and D. Vigh, 2012, A level set approach to salt geometry inversion in full-waveform inversion, in SEG Technical Program Expanded Abstracts 2012: Society of Exploration Geophysicists, 1-5.

Nguyen, B. D., and G. A. McMechan, 2013, Excitation amplitude imaging condition for prestack reverse-time migration: Geophysics, 78, S37-S46.

Shen, X., I. Ahmed, A. Brenders, J. Dellinger, J. Etgen, and S. Michell, 2018, Full-waveform inversion: The next leap forward in subsalt imaging: The Leading Edge, 37, no. 1, 67b1-67b6.

Tarantola, A., 1984, Inversion of seismic reflection data in the acoustic approximation: Geophysics, 49, no. 8, 1259-1266.

Virieux, J., and S. Operto, 2009, An overview of fullwaveform inversion in exploration geophysics: Geophysics, 74, no. 6, WCC1-WCC26.

Wu, R.-S., and G.-X. Chen, 2018, Multi-scale seismic envelope inversion using a direct envelope fréchet derivative for strong-nonlinear full waveform inversion: arXiv preprint arXiv: 1808.05275.

Yang, Y., and B. Engquist, 2017, Analysis of optimal transport and related misfit functions in FWI: Geophysics, 83, no. 1, $1-25$. 